\title{
Developing intentionality and researching multilingually: an ecological and methodological perspective
}

\author{
$3^{\text {rd }}$ resubmission to 'Researching Multilingually' special issue of the \\ International Journal of Applied Linguistics
}

Juup Stelma and Richard Fay

School of Education, The University of Manchester

\author{
Xiaowei Zhou \\ Business School, Edinburgh Napier University
}

\begin{abstract}
This article uses an ecological perspective to understand a researcher's developing intentionality vis-à-vis her multilingual engagement with research processes - i.e. 'researching multilingually'. The article discusses ecological thinking in Applied Linguistics, ecological themes present in the literature addressing aspects of researching multilingually, and the particular understanding of intentionality we have employed. The potential of intentionality as a heuristic for understanding researching multilingually is explored with reference to a recent research experience of one of the authors - Xiaowei Zhou. This includes analyses of Xiaowei's developing awareness of linguistic possibilities, associated researchrelevant affordances, and her articulation of the intentionality of researching multilingually. We suggest that the combination of an ecological perspective and a focus on intentionality provides a unique methodological understanding of researching multilingually.
\end{abstract}

\section{Key words:}

Researching multilingually; Research methodology; Intentionality; Ecological perspective; Research education.

\section{Introduction}

This article uses an ecological perspective to understand a researcher's developing 'intentionality' vis-à-vis 'researching multilingually'. We understanding 'intentionality' akin to the folk-psychological meaning of 'being purposeful' (Bruner 1981; Dennett 1987). By 'researching multilingually', we mean research - with or without an explicit focus on language and/or multilingualism - in which researchers engage multilingually with, inter alia, research literatures and research processes (including informed consent/ethics processes, data generation, data processing/analysis, and dissemination of research findings).

The article begins with a discussion of ecological work in Applied Linguistics, including also how this ecological view is represented in literature addressing different aspects of researching multilingually. We then introduce our particular definition and use of the concept of intentionality. Next, we use a recent research experience of one of the authors - Xiaowei Zhou - to exemplify the development and articulation of intentionality underpinning 
researching multilingually. We conclude with a discussion of the conceptual and methodological contributions, of our combination of an ecological perspective and the focus on intentionality, to understanding researching multilingually.

\section{Ecological thinking, Applied Linguistics, and researching multilingually}

Ecological thinking in Applied Linguistics reflects both a variety of intellectual influences and the many unique situations of language use being investigated. One body of ecological literature appears influenced by Haugen's (1972) ecology of language perspective. This includes, among other, explorations of the emergence of English as a global language (Phillipson and Skutnabb-Kangas 1996), local and regional linguistic shifts attributable to post-colonial and globalising processes (Makoni, Brutt-Griffler and Mashiri 2007), and whether and how changes to the linguistic resources available to people affect human wellbeing and prosperity (MacPherson 2003). This literature recognises that 'languages, like living species, evolve, grow, change, live, and die in relation to other languages' (Hornberger 2002, p.33). In parts of this literature, there is an explicit valuing of linguistic diversity, suggesting that such diversity may provide people with more communicative affordances (Aronin and Singleton 2010). Some authors propose that with linguistic diversity people are better able to evolve solutions necessary for future human well-being and prosperity (MacPherson 2003).

Another strand of ecological thinking in Applied Linguistics draws on the sociolinguistic notion of linguistic repertoires (Gumperz 1973; Potter and Litton 1985). Blommaert (2009, p. 424) suggests that an individual's linguistic repertoire 'reflects a life, and not just birth, a life that is lived in a real sociocultural, historical and political space'. This focus on individuals' linguistic repertoires can be linked to the ecological argument for diversity. Martin-Jones and Saxena (2003) propose that the language repertoires of individuals give them access to 'funds of knowledge' which may be used to connect different linguistic contexts, practices and cultures. This view also adds nuance to the diversity argument. The opportunities for individuals or groups using any particular language to express meaning and to act in the world will depend also on the linguistic repertoires of interlocutors. For example, Aronon and Singleton (2010, p.116) suggest that 'the typological closeness of the Germanic Scandinavian languages and their shared historical and geographical context provide speakers of Norwegian, Danish and Swedish with more communicative affordances than are normally furnished for speakers of different languages'. The significance of broader linguistic environments is captured by Blommaert, Collins and Slembrouck (2005, p.197) when they argue that linguistic activity depends not only on 'what individuals have or don't have, but [also] what the environment, as structured determinations and interactional emergence, enables and disables'.

The ecological perspective on linguistic activity is evident also in literature addressing different aspects of researching multilingually. One ecological theme is the concern for how researchers' and participants' linguistics repertoires and experiential knowledge shape the ways in which data is generated, processed and interpreted. This theme is evident in discussions of multilingual interview scenarios. Cortazzi, Pilcher and Jin (2011, p.529) 
recommend considering 'the possibilities and implications of different language choices available' in an interview situation, and themselves provide a systematic analysis of qualitative variation between data generated with Chinese participants in English-medium versus Chinese-medium interviews. Temple and Edwards (2002) focus on the role of interpreters in multilingual interview situations, arguing for a reflexive approach that includes the perspectives that the interpreters bring to the research process. The theme also appears in discussions of translation of data, including here a broader concern with the experiential knowledge of translators (Shklarov 2007).

A second ecological theme is how language, as an emergent property of communities and cultures, may 'index' research participants' experience and identity. Bradby (2002) has looked at how languages constitute unique linguistic affordances for research participants, and how their language use may 'index' their identity, including 'generation, gender, local attachment, minority status and resistance to racism' (p. 852). Vinokurov, Geller and Martin (2007) have explored how this indexing quality of language affects translation as well as subsequent data analysis and interpretation. They propose that since translation involves moving participants' 'meanings' into a different linguistic, sociohistorical and cultural system - i.e. that of the translator or of the researcher - translation discrepancies may be used to explore how the different languages involved in the research represent meanings. Understanding a translation discrepancy may then be a used to reconstruct otherwise inaccessible participant meanings.

In a time when globalising processes exert a strong shaping influence on Universitybased research communities (Altbach, Reisberg and Rumbley 2009; Ishikawa 2009), the ecological insights offered by Applied Linguistics have clear implications. Researchers need to understand the potential impact on research outcomes of a single dominant language suppressing multilingual engagement in research processes. Conversely, there needs to be an awareness of the potential contribution of researchers with multilingual resources, and of researchers who frame their studies to include multilingual possibilities. However, harnessing the potential of linguistic opportunities in researching multilingually requires a strategic approach, and this is why we now turn to a discussion of intentionality in research.

\section{Research as intentional activity}

We understand intentionality akin to the folk psychological meaning of 'being purposeful' (Dennett 1987; see Searle 1983 for an alternative philosophically-based view on intentionality). We sometimes make this more overt with the phrase 'intentional action'. When we use the word 'intention' we make an interpretation about the cognitive state associated with intentionality (see Malle, Moses and Baldwin 2001). Bruner (1981, p.41) suggests that 'an intention is present when an individual operates persistently towards achieving an end state, persists in developing means and corrects the development of means to get closer to the end state, and finally ceases the line of activity when specifiable features of the end state are achieved'.

In previous research, we have explored the developing researcher intentionality of UKbased doctoral students (Stelma 2011) and Masters students (Stelma and Fay 2012). We see 
the focus on intentionality as enriching both our epistemology and our research practice. Our overt concern with intentionality helps us to keep the researcher's experience and development in focus. In our practice as researchers and research educators, the concern with intentionality helps us to identify when decisions about researching multilingually are governed by alternative 'impulses', such as fashion, convention or expedience. In this respect, we draw inspiration from the wider ecological literature which sees the meaning that we as individuals construct, and the intentions that we pursue, as the defining quality of human lived experience (Bang 2007; Heft 1989; Young, DePalma and Garrett 2002).

\section{Intentionality and researching multilingually}

The following discussion focuses on an experience of one of the authors of this article Xiaowei Zhou - of researching multilingually. The experience was Xiaowei's doctoral project (Zhou 2010), when she narratively explored the academic acculturation experiences of six students from mainland China during their first year of study in the UK. This focus on a single researcher's experience enables an in-depth and multi-voiced discussion of the development and articulation of intentionality underpinning researching multilingually. The multi-authored quality of the discussion represents a composite of Xiaowei contributing data and interpretation of her own experience, Richard being the driving force behind our researching multilingually thinking, and Juup adding the ecological conceptualisation and the focus on intentionality. Hence, the analysis is neither a 'data-driven' understanding of Xiaowei's experience, nor is it an 'application' of theory to data. Rather, the multiple perspectives of the authors, interacting with each other and with the data to generate understanding of researching multilingually, may be described as an ecological process in itself.

Following the work of Young et al. (2002), on the intersection of intentionality and affordance, we understand the intentionality of researching multilingually as evolving from an awareness of linguistic possibilities and their associated research-relevant affordances (see Stelma 2011). For this reason, we start by looking at Xiaowei's developing awareness of linguistic possibilities for her research before and upon arriving in the UK to start her doctoral studies. In this section, we rely on Xiaowei's retrospective reflections subsequent to completing her PhD. These were generated as part of her own work presenting on and publishing from her doctoral project (Fay, Zhou and Liu 2010; Zhou 2012). This is followed by a discussion of Xiaowei's exploration of the research-relevant affordances associated with different linguistic possibilities in her research. Here we make use of extracts from Xiaowei's PhD thesis, thereby exploring how Xiaowei articulates the developing intentionality of researching multilingually in her final $\mathrm{PhD}$ thesis.

\section{Developing awareness of linguistic possibilities}

Xiaowei's experience as a researcher started during her English-medium Masters studies at a University located in mainland China. In her Masters dissertation research, she interviewed participants in Mandarin Chinese and reported on her research in English. When she later looked back on this experience, she commented: 
As a student with a linguistic academic background ... my emerging awareness of "doing research bilingually" was mainly about translation issues: i.e. wherever I needed to present Mandarin data extracts in my research report, I translated the originals carefully by adding explanatory footnotes. I paid attention to the nuances between different word choices in the translation process and brought any interesting points into my discussion. Therefore, I considered the idea of "doing research bilingually" to be only relevant in the report write-up stage (Retrospective reflections 2010).

It appears then that Xiaowei's academic background in linguistics, and her high level of competence in both the Mandarin and English languages, combined to shape a particular awareness of the scope of researching multilingually; i.e. the careful approach to translation and representation.

When Xiaowei sent her application to do a $\mathrm{PhD}$ in the UK, wishing to focus on the academic sojourner experiences of Chinese students in the UK, her research proposal did not comment on the potential multilingual aspects of her project. Xiaowei's retrospectively commented that this was because her proposal did not address the thesis writing stage. However, she did describe how the move to the UK stimulated her further development as a researcher.

I believe that my personal development as a researcher took place in ... a complex of transfers, between China and UK, between the academic culture of my previous university and department and that of my present ones, between my studies at the Masters level and those at the Doctoral level, between my previous disciplinary home in language studies and my current one in social sciences ... (Retrospective reflections 2010).

Xiaowei also pointed out how this development 'corresponded to ... [her] ... PhD study on the academic acculturation experiences of students from China', thereby ecologically connecting her evolving research focus, the shared experience she had with her research participants, and her developing researcher identity.

Xiaowei's development included gradually raised awareness of a broader set of linguistic possibilities in her research on Chinese sojourner experiences. This aspect of her developing researcher competence was supported by her supervisor - Richard - and his encouragement for Xiaowei to join a UK network of researchers interested in researching multilingually (see Researching Multilingually Website: http://www.researchingmultilingually.com). As the following analysis will show, Xiaowei developed a comprehensive approach to the multilingual aspects of her doctoral research. The following represents the areas of her $\mathrm{PhD}$ project which were shaped by her developing awareness of multilingual possibilities in research:

- Contextualisation of the research in both China and the UK. 
- Conceptualisation using both English-medium and Chinese-medium literature.

- Data generation, including consideration of participants' linguistic resources and Chinese/English-informed narrative-inducing techniques.

- Data processing, including developing a transcription protocol for the Chinese data.

- Data analyses and interpretation drawing on English-medium and Chinese-medium experiences.

- Thesis write-up, including translation issues, transparency and reflexivity.

We see this expanded awareness of linguistic possibilities as integral to Xiaowei eventually developing an expanded set of intentionalities to underpin researching multilingually.

\section{Exploring multilingual affordances}

In this section, we make reference to Xiaowei's $\mathrm{PhD}$ thesis text to understand her exploration of research-relevant affordances associated with the various linguistic possibilities of which she was becoming aware. We begin with Xiaowei's exploration of how the concepts of 'culture' and 'intercultural' were developed in the Mandarin-medium literature.

As I set out on my study and realised the potential advantage of my bilingual background, I began to also explore relevant literature written in Mandarin. First, I examined the contemporary Mandarin-medium literature on文化，the Mandarin equivalent for culture ... I ... noticed that the [Mandarin] writers tend to associate culture with countries or larger geographical entities ... As I preferred small-culture understandings of culture, I did not find this literature particularly insightful for my study ... Next, I examined the Mandarin-medium literature on 跨文化交际， the Mandarin equivalent for intercultural communication. Through this examination, I realised that the academic field, including the concept, of intercultural communication was imported by some Mandarin-speaking scholars from the English-medium academia to that in mainland China (Zhou 2010, p. 31).

Xiaowei's exploration of the Mandarin-medium literature may be described as a search for research-relevant affordances. She had the necessary linguistic repertoire to establish that the Mandarin-medium literature did not help move forward the conceptualisation of her area of research. However, her thesis does recognise and refer to the Mandarin-medium literature, thereby acting to make this literature more visible. Moreover, as will become clear below, Xiaowei's exploration of the Mandarin-medium literature benefited later stages of her research.

A contrasting case is offered by the linguistic possibilities that presented themselves during the data coding and interpretation stages of Xiaowei's $\mathrm{PhD}$ research project. The 'raw' data available to Xiaowei was mainly Mandarin-medium interview data (we discuss the multilingual dimension of Xiaowei's data generation in the next section). The following extract from her thesis reflects the exploration of linguistic affordances for the data coding and interpretation stages: 
I planned to allow an interplay between Mandarin and English in my data exploration, e.g. to code themes using concepts which were original expressions used by my participants or concepts which I learnt from relevant literature written in Mandarin or in English. I also planned to draw on my Mandarin/English-medium personal experiences in my data exploration and interpretations, e.g. to note interesting points in my participants' personal experience stories relating to their previous academic experiences in mainland China and the current challenges they encountered in their academic lives in the UK, points which might resonate with my own academic experiences in both countries (Zhou 2010, p.124-125).

The above extract describes a number of linguistic possibilities that came together at this stage of the research. Among others, we see how Xiaowei's earlier exploration of the Mandarin-medium literature now takes on new relevance. The following extract illustrates how the linguistic repertoires and background that Xiaowei shared with a participant aided her interpretation of this participant's experience of acculturation in the UK university setting.

Almost every time when I saw him, he was on his way to, or back from, his school or the library, with a bag on his shoulders and course materials in his hands. This impression evoked in my mind the typical image of what many students consider to be a "good student" in mainland China ... George's own comment on his academic life somehow resonated with my impression above. He considered his academic life to be as simple as “三点一线 / san dian yi xian [three points on a line]", the three points being the lecture rooms, library, and the residence hall. (Zhou 2010, p.228).

三点一线 / san dian yi xian [three points on a line] is a common Chinese expression used to complain or joke about a life situation which is very basic, or 'too basic'. Whilst a similar meaning may be expressed in English (e.g. 'eat, sleep and work'), the metaphoric aspect of 'three points on a line' adds poignancy. Given the relative ubiquity of this experience in Chinese society, the linguistic expression may then be understood as a conceptual metaphor (Lakoff and Johnson 1980) which Xiaowei shares with her participant. In other words, Xiaowei's embodied knowledge of student life in China makes her uniquely attuned to the feelings and other associations that George evokes by using this expression. This, then, represents a linguistically constituted affordance that Xiaowei pursued to strengthen the credibility of her researcher interpretations.

\section{Developing and articulating intentionality}

Developing awareness of linguistic possibilities in research, and the exploration of the research-relevant affordances of these possibilities, are both part of the process of developing intentionality. In this section, we look at intentionality more directly. We explore how Xiaowei develops and articulates the intentionality of researching multilingually in her $\mathrm{PhD}$ thesis. This analysis recognises that intentionality may manifest itself differently in writing. 
We need to add that while writing her $\mathrm{PhD}$ thesis Xiaowei was not using intentionality as a heuristic for understanding the multilingual dimension of her research.

The methodology chapter of the thesis, where she introduces the qualitative/narrative approach of her doctoral project, is perhaps where Xiaowei most clearly articulates intentionality. In this chapter, she uses overt means-end statements, or 'reason explanations'. This is a conceptualisation of intentionality prevalent in cognitive and developmental psychology (see Malle, Moses and Baldwin 2001). We start by exploring this overt articulation of intentionality, and subsequently relate this to what she says about researching multilingually. Here, we underline the words/phrases in Xiaowei's thesis (Zhou 2010, pp.125-126) from which we infer intentionality.

a. 'I aimed to make plausible and justifiable interpretations of my participants' subjectively-constructed academic experiences.'

b. 'to enhance the trustworthiness of my study, I planned to keep interrogating myself about my positions, choices, and actions throughout my conceptualisation of the study ... research design, data generation, data processing, data exploration, and data interpretation.'

c. 'I would write up my research report as transparently as I could ... so that readers would be able to see how my study developed under the influences of my positions, interest, and preferences.'

The above may be understood as means-end statements providing reason explanations for Xiaowei's research activity. That is, Xiaowei may be seen as acting with intention in the sense that her actions have an 'end'. This is most visible in the second and third points above, which can be re-presented as follows:

I planned to keep interrogating myself (means) to enhance trustworthiness (end)

I would write transparently (means) so that readers would see how my study developed (end)

However, this is not an ecological account of intentionality until we recognise that, and explore how, these 'ends' may be intentional as well. This requires us to consider the research community that Xiaowei was becoming a part of. Different research communities tend to have particular expectations about how to conduct research, and the intentionality of these expectations are developed in bodies of research literature. For example, rationales for trustworthiness and transparency are developed in the qualitative research literature (e.g. Lincoln and Guba 1985). Given the overt references to such expectations as trustworthiness and transparency, we recognise that Xiaowei's research activity, and her developing intentionality as a researcher, may have been shaped by expectations that she perceived to be present in the broader qualitative research community she was becoming a part of. That is, an ecological understanding of researcher intentionality recognises the co-adaptive relationship 
of purposes developed by the individual and purposes codified as expectations evident in research communities.

In terms of ethics, Xiaowei distances herself from what she perceives to be the expectations of the qualitative research community. She cites Robinson-Pant (2005) in support of the suggestion that 'ethical guidelines set by Western or UK academic institutions may not ... cover concerns arising for researchers undertaking bilingual research' (Zhou 2010, p.127). Drawing on Bassey (1999), she concludes:

d. 'I decided to address the ethical aspect of my study through a general principle of "respect for persons", (Zhou 2010, p.127).

Next, we take a closer look at whether and how what Xiaowei said about researching multilingually relates to the mentioned expectations in the qualitative research community, as well as how decisions about researching multilingually related to her generally stated intention about how to approach ethics in her research. We start with Xiaowei's rationale for using Mandarin as the language of her interviews.

I believed that Mandarin would help [a] my participants fluently re-construct their experiences ... [b] establish a comfortable atmosphere for talk and [c] an insiderinsider relationship between me and my participants. Nevertheless, [d] I would respect my participants' will if they preferred to use English (Zhou 2010, p.124).

In this extract, [a], [b] and [c] articulate research-relevant affordances of using Mandarin as the language of the interviews, and [b] and [d] articulate the particular concern for ethics in the research. The following introduces our interpretation of the underlying intentionality of this articulation.

[a] using Mandarin enhances the trustworthiness of the data.

[b] establishing a comfortable atmosphere contributes to the general principle of respect for persons, and thereby enhances the trustworthiness of the data.

[c] the insider-insider relationship enables me to make plausible and justifiable interpretations of the participants' subjectively-constructed academic experiences, and thereby enhances the trustworthiness of the data.

[d] respecting my participants linguistic preferences contributes to the general principle of respect for persons.

Provided our analysis, including Xiaowei herself as a co-analyst, is a plausible interpretation of the thesis text, Xiaowei's researching multilingually appears ecologically linked to broader expectations about how researchers may enhance the trustworthiness of qualitative data - in [a], [b], and [c]. Add to this, Xiaowei's rationale appears to be shaped by her ethics-based intentionality for deciding what language to use in an interview - in [b] and [d].

Another aspect of researching multilingually with overtly articulated intentionality is Xiaowei's discussion of decisions about language representation in preparing translations and 
writing her final thesis. In this case she foregrounds rigour and transparency, and these may also be understood as expectations of the research community with which she was engaging.

While translating the extracts, I would check the transcripts carefully so as to ensure that I found the best possible equivalents in English. I would also pay attention to any subtle meanings I identified from the extracts, and work towards a presentation of them as accurately as possible through my careful considerations and perhaps discussions with my bilingual friends ... I would provide these extracts to the reader in both their original and translated versions so that s/he may have a chance to compare my translations with their originals (Zhou 2010, p.125).

The underlined subordination and coordination devices appear to justify her micro-level decisions about representation, and may therefore be understood as an overt articulation of intentionality. Xiaowei also makes transparent the role of her own, her bilingual friends', as well as her readers' language repertoires in the intentionality under-pinning her decisions about representation.

As part of our exploration of the means-end articulation of intentionality in Xiaowei's thesis, we expected the multilingual engagement with research processes to be presented as a means by which an overall research aim was being achieved. As we explored this relationship, we were struck by a different quality of Xiaowei's development and articulation of intentionality. At the start of her PhD thesis, Xiaowei introduces her research as follows:

In broad terms, my study focused on the academic experiences of some students from mainland China studying in the United Kingdom ... This focus built on my interest in how individuals communicate with other individuals from different cultural backgrounds and how they come to terms with cultural contexts that are in some ways foreign to them. My own experiences of encountering difference or foreignness both in mainland China and in the UK ... prompted this research interest and provided personalised experience of it (Zhou, 2010, p.16).

Noteworthy in this extract, as well as in the remainder of the introductory chapter, is the absence of an overtly stated research aim. Instead, Xiaowei's introduction expands on the above paragraph by providing a personal narrative, thereby framing her $\mathrm{PhD}$ work as growing out of her own past experience and her developing academic interest. Given that this narrative style extends to other parts of Xiaowei's thesis (although more evident in some parts than others) we wondered whether her style of writing might have affected how intentionality was developed and articulated. In terms of our analytical approach, we wondered whether the means-end view of intentionality should be challenged as our only view on how intentionality was articulated in Xiaowei's thesis. Using the intersection of narrative and intentionality as a new 'lens' on Xiaowei's writing, we formulated three hunches about different ways in which intentionality might develop and be articulated, not only in Xiaowei's text, but perhaps also in research writing more generally. 
Our first hunch is based on the suggestion, by Herman (2008, p. 249), that narrative is both an expression of intentionality as well as 'a semiotic environment in which humans learn to take up the intentional stance in the first place'. Herman's view suggests that Xiaowei's narrative account may at the same time be grounded in her developing intentionality and be the semiotic environment that gives rise to her intentionality. This leaves open the possibility that some aspects of intentionality may be more clearly developed and articulated in a research text; in Xiaowei's thesis, this seems to apply to the ecological relationship between her decisions about researching multilingually and the expectations she perceives to be present in the qualitative research community. Conversely, other aspects may be less clearly and/or less overtly articulated; in Xiaowei's case the relationship between researching multilingually and her overall research aim.

Our second hunch is based on Bruner's (1991, p.4) argument that narratives 'are a version of reality whose acceptability is governed by convention and "narrative necessity" rather than by empirical verification and logical requiredness'. Bruner implies that 'narrative necessity' arises when there is a deviation from a canonical cultural pattern. The innovative methodological practices described in this special issue on researching multilingually may perhaps be understood as deviation from the canonical practices of the research community. Moreover, Bruner views intentionality as a feature of narrative, and he suggests that when a protagonist does something out of the ordinary - i.e. deviates from a canonical pattern of behaviour - the intentional state of the protagonist may be evoked to create a reason for the action. From this, we suggest that when research texts account for well known or conventional research events and practices there may be less of an expectation for, or need to, overtly articulate intentionality. Conversely, when a research text grapples with comparatively novel research events or practices, such as are involved when researching multilingually, there may be more of a 'necessity' to overtly articulate intentionality.

Our third hunch arises from Malle's (2001) review of different ways that intentionality may be developed and articulated. In addition to the more common 'reason explanations' (i.e. means-end statements), Malle suggests that the articulation of intentionality may include, among others, 'causal history of reasons' explanations. Looking at the above quote from Xiaowei's introductory chapter, the following sentence seems to be a 'causal history of reasons' explanation: 'My own experiences of encountering difference or foreignness both in mainland China and in the UK ... prompted this research interest'. More generally, Xiaowei's narrative account of her developing interest in sojourner experiences in her introductory chapter might then be classed as an extended 'causal history of reasons' explanation, and the cumulative effect of this narrative writing may be understood as an articulation of intentionality.

Finally, we would like to introduce another quality of intentionality that we experienced in co-authoring this article. Gibbs (2001, p.122) suggests that 'many aspects of intentional meaning ... are products of dynamic social interactions and not solely the result of privately held, internalized mental representations'. Gibbs makes the further suggestion that when intentionality is understood as dynamical and interactive, the role of others (listeners, readers and observers) in shaping intentional meaning and action may be considerable. We note again that until the writing of this article Xiaowei was not overtly using intentionality as a heuristic 
when considering multilingual possibilities and affordances in her research. We suggest, then, that despite having Xiaowei onboard as a co-author and an 'insider' interpreter, the intentionality we now 'see' in her thesis text may include both: a) a reproduction of intentionality that Xiaowei was aware of when she wrote her thesis, and b) intentionality which we 'discovered' in the text while working together to write the present article. We see this as a reflection of the rich 'potential of text' - of how the intentionality of any text is first articulated by a writer, and then (re)interpreted by successive reading and re-reading.

\section{Conclusions}

In this conclusion, we wish to highlight again our ecological understanding of developing intentionality in researching multilingually, what we think we are learning about the articulation of intentionality in research writing - including the particular observations made about intentionality and narrative - and finally what we believe are the methodological implications for researchers encountering research situations with multilingual potential.

We have suggested that researching multilingually is ecologically shaped by researchers' reflections on their own and others' linguistic repertoires and associated experiential knowledge, as well as the parallel engagement with expectations in the research community. Our analysis of Xiaowei's researching multilingually shows how her exploration of linguistic possibilities was enabled by her own linguistic repertoire and experiential knowledge, as well as her awareness of her participants' linguistic repertoires and experiential knowledge. Moreover, the analysis reveals how Xiaowei's exploration of research-relevant linguistic affordances, and her parallel developing intentionality, was ecologically shaped by, and in her writing related to, expectations about trustworthiness and transparency that she perceived to be present in the qualitative research community. More particularly, we have demonstrated this ecological understanding with reference to Xiaowei's development and articulation of the intentionality shaping her use of Mandarin as the language of her narrative interviews and her decisions about language representation in preparing translations and writing her final thesis.

We have also explored how the intentionality underpinning researching multilingually is developed and articulated in Xiaowei's thesis text. This analysis indicates that intentionality may be articulated in various ways in writing. The most clearly visible intentionality in Xiaowei's thesis appears as overt reason explanations, or means-end statements. She used such statements to link her decisions about multilingual aspects of her research to the shared expectations about trustworthiness and transparency she perceived to be present in the qualitative research community. We also identified a narrative structuring of Xiaowei's thesis text, and explored three ways in which intentionality might relate to narrative ways of thinking and writing. This part of the discussion offers particular insights for narrative research and writing. Also, this discussion suggests a need to remain open to different explanations in our further work on understanding the development and articulation of intentionality in researching multilingually, as well as research activity more generally.

Finally, we suggest that our exploration of Xiaowei's experience of researching multilingually, and how she articulated the intentionality of her research activity in her thesis, may be used as a framework to guide researcher reflection when encountering research 
situations that may require multilingual engagement with research processes. Becoming aware of the potential diversity of linguistic possibilities in research with multilingual dimensions seems both prerequisite and integral for developing researcher intentionality. Xiaowei's experience illustrates that a reflexive engagement with one's own linguistic repertoire and associated experiential knowledge may aid in developing this kind of awareness (see Bashiruddin this issue for additional exploration of this area). An exploration of the research-relevant affordances of different linguistic possibilities is a further move towards developing intentionality while researching multilingually. Also crucial is the recognition that intentionality is ecologically constituted. It requires researchers' engagement with expectations present in the research community they are becoming a part of. Finally, researchers may benefit from exploring different ways of articulating their developing intentionality when writing about researching multilingually.

Text in this resubmission is $\mathbf{5 3 8 5}$ words (not counting title, abstract and references).

\section{References}

Altbach, P.G., Reisberg, L., and Rumbley, L.E. (2009). Trends in global higher education: Tracking an academic revolution. A Report Prepared for the UNESCO 2009 World Conference on Higher Education. Paris: UNESCO.

Aronin, L. and Singleton, D. (2010). Affordances and the diversity of multilingualism. International Journal of the Sociology of Language, 205: 105-129.

Bang, J. (2007). Steps towards an ecological approach to thinking. Anthropological Psychology, 18: 2-13.

Bassey, M. (1999). Case study research in educational settings. Buckingham: Open University Press.

Blommaert, J. (2009). Language, Asylum, and the National Order (with comments by various authors). Current Anthropology, 50(4): 415-441.

Blommaert, J., Collins, J., and Slembrouck, S. (2005). Spaces of multilingualism. Language and Communication, 25: 197-216.

Bradby, H. (2002). Translating culture and language: a research note on multilingual settings. Sociology of Health and Illness, 24(6): 842-855.

Bruner, J.S. (1981). Intention in the structure of action and interaction. In L. Lipsitt (ed.) Advances in infancy research, Volume 1 (pp. 41-56). Norwood, NJ: Ablex.

Bruner, J. (1991). The narrative construction of reality. Critical Inquiry, 18(1): 1-21.

Cortazzi, M., Pilcher, N., and Jin, L. (2011). Language choices and 'blind shadows': investigating interviews with Chinese participants. Qualitative Research, 11(5): 505 535.

Dennett, D. (1987). The intentional stance. Cambridge, MA: MIT Press. 
Fay, R., Zhou, X., and Liu, T-H. (2010). Undertaking narrative inquiry bilingually against a monolingual backdrop. Paper presented at the conference "Narrative Matters 2010 Exploring the Narrative Landscape: Issues, Investigations, and Interventions", May 20th - 22nd 2010, Fredericton, Canada.

Gibbs, R. (2001). Intentions as emergent products of social interactions. In B.F. Malle, L.J. Moses, and D.A. Balwin (eds.) Intentions and intentionality: foundations of social cognition (pp. 105-122). Cambridge, MA: Bradford/MIT.

Gumperz, J.J. (1973). The communicative competence of bilinguals: some hypotheses and suggestions for research. Language in Society, 1(1): 143-154.

Haugen, E.I. (1972). The ecology of language. Stanford, CA: Stanford University Press.

Heft, H. (1989). Affordances and the body: An intentional analysis of Gibson's ecological approach to visual perception. Journal of the Theory of Social Behaviour, 19(1): 1-30.

Herman, D. (2008). Narrative theory and the intentional stance. Partial Answers: Journal of Literature and the History of Ideas, 6(2): 233-260.

Hornberger, N.H (2002). Multilingual language policies and the continua of biliteracy: an ecological approach. Language Policy, 1: 27-51.

Ishikawa, M. (2009). University rankings, global models, and emerging hegemony: critical analysis from Japan. Journal of Studies in International Education, 13(2), 159-173.

Lakoff, G. and Johnson, M. (1980). Metaphors we live by. Chicago: University of Chicago Press.

Lincoln, Y.S. and Guba, E.G. (1985). Naturalistic inquiry. Newbury Park, CA: Sage.

MacPherson, S. (2003). TESOL for biolinguistic sustainability: the ecology of English as a Lingua Mundi. TESL Canada Journal, 20(2): 1-22.

Makoni, S., Brutt-Griffler, J. and Mashiri, P. (2007). The use of "indigenous" and urban vernaculars in Zimbabwe. Language in Society, 36: 25-49.

Malle, B.F. (2001). Folk explanations of intentional action. In B.F. Malle, L.J. Moses, and D.A. Balwin (eds.) Intentions and intentionality: foundations of social cognition (pp. 265-286). Cambridge, MA: Bradford/MIT.

Malle, B.F., Moses, L.J., and Balwin, D.A. (2001). Introduction: the significance of intentionality. In B.F. Malle, L.J. Moses, and D.A. Balwin (eds.) Intentions and intentionality: foundations of social cognition (pp. 1-24). Cambridge, MA: Bradford/MIT.

Martin-Jones, M., and Saxena, M. (2003). Bilingual resources and 'funds of knowledge' for teaching and learning in multi-ethnic classrooms in Britain. International Journal of Bilingual Education and Bilingualism, 6(3\&4): 267-282.

Phillipson, R. and Skutnabb-Kangas, T. (1996). English only worldwide or language ecology? TESOL Quarterly, 30(3): 429-452. 
Potter, J. and Litton, I. (1985). Some problems underlying the theory of social representations. British Journal of Social Psychology, 24: 81-90.

Researching Multilingually Website (no date). http://www.researchingmultilingually.com.

Robinson-Pant, A. (2005). Cross-cultural perspectives on educational research. Maidenhead, Berkshire: Open University Press.

Searle, J.R. (1983). Intentionality: an essay in the philosophy of mind. Cambridge: Cambridge University Press.

Shklarov, S. (2007). Double vision uncertainty: the bilingual researcher and the ethics of cross-language research. Qualitative Health Research, 17(4): 529-538.

Stelma, J. (2011). An ecological model of developing researcher competence: the case of software technology in doctoral research. Instructional Science, 39(3): 367-385.

Stelma, J. and Fay, R. (2012). Intentionality and developing researcher competence on a UK masters course: an ecological perspective on research education. Studies in Higher Education. DOI: 10.1080/03075079.2012.709489.

Temple, B. and Edwards, R. (2002). Interpreters/translators and cross-language research: reflexivity and border crossings. International Journal of Qualitative Methods, 1(2): 112.

Vinokurov, A., Geller, D., and Martin, T.L. (2007). Translation as an ecological tool for instrument development. International Journal of Qualitative Methods, 6(2): 40-58.

Young, M.F., A. DePalma, and S. Garrett. (2002). Situations, interaction, process and affordances: an ecological psychology perspective. Instructional Science, 30(1): 47-63.

Zhou, X. (2010). A narrative exploration of the UK academic acculturation experience of students from mainland China. $\mathrm{PhD}$ thesis, University of Manchester, Manchester.

Zhou X. (2012). "Translating" insights into practice across languages: the case of a narrative study. Paper presented at the AHRC Researching Multilingually Seminar, University of the West of England, April $25^{\text {th }}-26^{\text {th }} 2012$, Bristol, UK. 